\title{
PAIRS OF INTERSECTING REAL MANIFOLDS IN COMPLEX SPACE *
}

\section{S. M. WEBSTER ${ }^{\dagger}$}

Introduction. In this work we consider two real $n$-dimensional submanifolds of complex $n$-space which intersect at the origin,

$$
0 \in M_{1} \cap M_{2}, \quad M_{1}, M_{2} \subset \mathbf{C}^{n} .
$$

We shall usually assume that the $M_{i}$ are totally real, and that the intersection is isolated and transverse, though other cases are eventually interesting. We are primarily interested in the holomorphic equivalence problem, i. e. to find a biholomorphic map fixing the origin and taking $M_{1}$ and $M_{2}$ simultaneously into some canonical form, but we also consider some specific geometric questions. For example, do there exist complex analytic curves through 0 and cutting each $M_{i}$ in a real curve? Do there exist analytic annuli in $\mathbf{C}^{n}$ with one bounding circle on $M_{1}$ and the other on $M_{2}$ ? What is the precise local hull of holomorphy of $M_{1} \cup M_{2}$ near 0 ?

The study of a pair of intersecting real curves in the complex plane was perhaps begun by E. Kasner in 1912, see Pfeiffer [6]. The case of two totally real linear $n$-planes in $\mathbf{C}^{n}$ was studied by Weinstock [12].

We shall consider three main cases: the generic case, the real Lagrangian case, and the holomorphically reversible case. By the generic case we shall mean that there is no additional structure imposed. In the real Lagrangian case we consider such real submanifolds $M_{i}^{2 n}$ in $\mathbf{C}^{2 n}$ with its (standard) holomorphic symplectic form $\omega$, where $\operatorname{Re}(\omega)$ vanishes when restricted to each $M_{i}^{2 n}$. The pair is holomorphically reversible if there exists a holomorphic involution $\tau$ near 0 , with $\tau M_{1}=M_{2}$.

In this paper we shall assume that $M_{1}$ and $M_{2}$ are real analytic, and use power series methods. Each $M_{i}$ is locally the fixed point set of an anti-holomorphic involution $\rho_{i}$

$$
M_{i}=F P\left(\rho_{i}\right), \quad \sigma=\rho_{1} \rho_{2} .
$$

We shall focus on the holomorphic normalization of the pair $\rho_{1}, \rho_{2}$, and then derive results about $M_{1}, M_{2}$. The holomorphic map $\sigma$, which will be central to our study, is anti-holomorphically reversible (briefly, anti-reversible), in that it is conjugate to its inverse by an anti-holomorphic involution:

$$
\sigma^{-1}=\rho_{2} \rho_{1}=\rho_{1}^{-1} \sigma \rho_{1} .
$$

The square $\sigma^{2}$ measures the extent to which $\rho_{1}$ and $\rho_{2}$ fail to commute. The above mentioned "cutting curves" $C$ satisfy $\rho_{1} C=\rho_{2} C=C$. We now have the additional concept of a pair of "switched curves", $\rho_{i} C_{1}=C_{2}, i=1,2$. Both are then invariant by $\sigma$.

In the holomorphically reversible case,

$$
\rho_{2}=\tau \rho_{1} \tau, \quad \sigma=\tau_{1} \tau, \quad \tau_{1}=\rho_{1} \tau \rho_{1},
$$

\footnotetext{
*Received November 2, 2002; accepted for publication June 3, 2003.

${ }^{\dagger}$ Department of Mathematics, University of Chicago, 5734 S. University Avenue, Chicago, Illinois 60637, USA (webster@math.uchicago.edu).
} 
which says that $\sigma$ is also the product of two holomorphic involutions. A special case of this was the key to the theory of analytic real $n$-manifolds in $\mathbf{C}^{n}$ with nondegenerate complex tangents developed in [5]. In the real Lagrangian case we have

$$
\rho_{i}^{*} \omega=-\bar{\omega}, \quad \sigma^{*} \omega=\omega, \quad \omega=\sum_{j=1}^{n} d z_{j} \wedge d z_{n+j} .
$$

We emphasize that we are using a holomorphic symplectic form, and not the more usual real part of the Kähler form.

Section 1 is concerned with the linear case. The eigenvalues of a linear antireversible map $\sigma$ occur in pairs. A linear transformation which diagonalizes $\sigma$ also takes the $\rho_{i}$ into the appropriate canonical form. Section 2 treats the non-linear case as an analytic or formal power series perturbation. A procedure is given (prop 1) to derive certain properties of $\rho_{1}, \rho_{2}$ from those of $\sigma$, under certain "non-resonance" conditions on the linear part of $\sigma$.

In section 3 we collect some known results on convergence relative to a general map $\sigma$ and adapt them to our needs here. In particular, theorem (2) gives an existence result for invariant submanifolds with linearization for $\rho_{1}, \rho_{2}$ in the generic case. Proposition (2) gives a convergence result for an invariant submanifold without linearization for a symplectic map $\sigma$, in the "integrable" hyperbolic case. The argument parallels that for flows given in [9].

In section 4 we derive symplectic (or unimodular) normal forms for anti-reversible symplectic maps $\sigma$ in $\mathbf{C}^{2}$. The theory splits into two cases (see (1.12)) according to whether the eigenvalues of $\sigma$ are (i) unimodular (4.5), (4.6), (4.9); or (ii) real (4.12), (4.13). In general these normal forms yield implicit normal forms for the real Lagrangian surfaces $M_{1}, M_{2}$. We also give an application to the local hull of holomorphy of $M_{1} \cup M_{2}$ in the higher dimensional real Lagrangian case.

Section 5 shows that our pair of real Lagrangian surfaces in $\mathbf{C}^{2} \ni(z, p), \omega=$ $d p \wedge d z$, may be given in terms of two analytic real functions $r_{i}$ as

$$
\begin{array}{ll}
M_{1}: & p=\partial_{z} r_{1}, \quad r_{1}=a z^{2}+b z \bar{z}+\overline{a z}^{2}+\cdots \\
M_{2}: & p=\partial_{z} r_{2}, \quad r_{2}=z \bar{z}+\cdots
\end{array}
$$

where $b \neq 0$, and $(a, b) \neq(0,1)$, and the dots represent terms of order three or higher. In case (ii) of (1.12), we apply the normal form for the involutions $\rho_{i}$ to write $(0.6)$ with functions $r_{i}$ of the form (see (4.16), (4.17) below)

$$
r_{i}=\lambda_{i}^{-1}|z|^{2}+\hat{r}_{i}\left(|z|^{2}\right), \quad \hat{r}_{i}(s)=O\left(s^{2}\right), \quad i=1,2
$$

where

$$
\lambda_{i}=\bar{\lambda}_{i} \neq 0, \pm 1, \quad \lambda_{1} \lambda_{2}=\epsilon= \pm 1 \text {. }
$$

One consequence of the results of sections 4 and 5 is the following.

Theorem 1. Suppose that

$$
\frac{b^{2}-4|a|^{2}+1}{2 b}>1 \text { in }(0.6), \text { or } \epsilon=+1 \text { in }(0.8) \text {. }
$$

Then there exists a real analytic 1-parameter family of analytic annuli $A_{c} \subset \mathbf{C}^{2}$, $0<c<c_{0}$, bounding on $M_{1} \cup M_{2}$, and shrinking to the origin as $c \rightarrow 0$. These annuli 
sweep out a three dimensional Levi-flat manifold lying on a real analytic set, which is biholomorphic to $\operatorname{Im}(z p)=0$, with $\operatorname{Re}(z p)>0$.

The moduli of these annuli determine to large extent the invariants of the pair of surfaces (see (4.19) below). The union of these annuli $A_{c}$ contribute to the local holomorphic hull of $M_{1} \cup M_{2}$. It is perhaps interesting to note the instability inherent in this result. If either $M_{1}$ or $M_{2}$ is slightly perturbed so as to destroy the real Lagrangian condition, then all these analytic annuli may be lost, and $M_{1} \cup M_{2}$ may actually become holomorphically convex. This is reminiscent of fixed point results for area preserving mappings [9], which may be lost under generic perturbation.

A holomorphically reversible analogue of the foregoing is contained in [5]. In fact, under certain conditions on the tangent planes to the $M_{i}$ at the origin, the holomorphic involution $\tau$ is the covering involution of a 2 -fold branched cover which maps $M_{1} \cup M_{2}$ to a real surface $N$ with elliptic complex tangent in $\mathbf{C}^{2}$. The holomorphic normal form for $M_{1}, M_{2}$ yields the normal form for $N$. That the corresponding annuli $A_{c}$ are mapped to the Bishop analytic discs [1] bounding on $N$ was first pointed out to the author by N. Sibony [8]. Furthermore, a global case of holomorphic reversibility occurs in [11] in the study of the Kobayashi extremal discs of an ellipsoidal domain. Therefore, we have concentrated here on the real Lagrangian case. A preliminary study indicates that theorem one should also hold in the smooth case, by a direct construction of analytic annuli. This will be taken up in a future work.

$\mathrm{X}$. Gong has recently shown that there exist anti-reversible maps $\sigma$ which are formally holomorphically reversible, but that the involution $\tau$ cannot have a positive radius of convergence [3]. Thanks are due to him for discussions leading up to this work.

1. The linear theory. In this section we consider the relevant aspects of a pair of linear $n$-planes $M_{1}, M_{2}$ passing through the origin of $\mathbf{C}^{n}$. With $M_{2}$ totally real and $M_{1}$ transverse to it, we may write

$$
\begin{aligned}
M_{2} & =\{y=0\}=\{z=\bar{z}\} \\
M_{1} & =\{x=A y\}=\{z=B \bar{z}\} \\
z & =x+i y, \quad \bar{z}=x-i y
\end{aligned}
$$

The two matrices $A, B$ are related by the Cayley transform [13], $\mathcal{C}: \mathcal{A} \rightarrow \mathcal{B}$,

$$
\begin{gathered}
B=\mathcal{C}(A)=(A+i I)(A-i I)^{-1} \\
A=\mathcal{C}^{-1}(B)=i(B+I)(B-I)^{-1} \\
\mathcal{B}=\{B \mid B \bar{B}=I\}, \mathcal{A}=T_{I} \mathcal{B}=\{A \mid A=\bar{A}\} .
\end{gathered}
$$

The "spectral mapping theorem" relates the eigenvalues,

$$
A v=\lambda v \Leftrightarrow B v=\mu v, \mu=\frac{\lambda+i}{\lambda-i}, \lambda=i \frac{\mu+1}{\mu-1} .
$$

In particular, $\lambda \mapsto \bar{\lambda}$ corresponds to $\mu \mapsto \bar{\mu}^{-1}$, the real line to the unit circle, and the imaginery axis to the real axis. If $M_{1}$ contains some non-zero $z$ and $i z$, then this gives $A$ an eigenvector to $\lambda= \pm i$, i. e. $\mu=0, \infty$. We exclude this case, so that $B$ 
is non-singular. Then $M_{1}, M_{2}$ are both totally real and are the fixed point sets of anti-linear involutions $\rho_{1}, \rho_{2}$,

$$
\rho_{1}(z)=B \bar{z}, \quad \rho_{2}(z)=\bar{z}, \quad \sigma(z)=\rho_{1} \rho_{2}(z)=B z .
$$

We briefly consider the case of two totally real $n$-planes with non-transverse intersection $M_{0}=M_{1} \cap M_{2}$. If again $\rho_{i}$ are the involutions, then

$$
M_{0}=\left\{z \mid \rho_{1}(z)=\rho_{2}(z)=z\right\} \subset\{z \mid \sigma(z)=z\} .
$$

Thus, $M_{0}$ is the fixed point set of $\rho_{1}(z) \equiv \rho_{2}(z)$ as acting on the +1 eigenspace of $\sigma$. Conversely, if this eigenspace is of positive dimension, we get such an $M_{0}$. The case $\operatorname{dim} M_{0}=n-2$ occurs in connection with a quadratic real $n$-manifold in $\mathbf{C}^{n}$ with a non-degenerate complex tangent [5].

a) Now we consider the generic case of the linear theory. Let $e$ be an eigenvector of $\sigma$ with eigenvalue $\mu$,

$$
\sigma e=\mu e, \Rightarrow \rho_{2} e=\bar{\mu} \rho_{1} e=\bar{\mu} \sigma \rho_{2} e .
$$

Thus, $\rho_{1} e$ and $\rho_{2} e$ are dependent eigenvectors of $\sigma$ with the eigenvalue $1 / \bar{\mu}$. We consider two cases,

$$
\text { (i) } \mu=\bar{\mu}^{-1}, \quad(i i) \mu \neq \bar{\mu}^{-1} .
$$

We make the assumption here and in general that all eigenspaces are one-dimensional.

In case (i) we have

$$
\rho_{i} e=\lambda_{i} e, \quad \lambda_{i} \bar{\lambda}_{i}=1, \quad i=1,2, \quad \mu=\lambda_{1} \bar{\lambda}_{2} .
$$

The complex line spanned by $e$ cuts each $M_{i}$ in a real line. If we make the change $e \mapsto c e$, then $\lambda_{i} \mapsto(\bar{c} / c) \lambda_{i}, i=1,2$. Thus, we may arrange either $\lambda_{2}=1$, or more symmetrically

$$
\lambda_{1} \lambda_{2}=1
$$

In case (ii) we set

$$
\begin{aligned}
\sigma e_{1}=\mu e_{1}, & \sigma e_{2}=\bar{\mu}^{-1} e_{2}, \\
\rho_{i} e_{1}=\lambda_{i} e_{2}, & \rho_{i} e_{2}=\bar{\lambda}_{i}^{-1} e_{1}, \quad \mu=\bar{\lambda}_{2} / \bar{\lambda}_{1} .
\end{aligned}
$$

The complex lines spanned by $e_{1}$ and $e_{2}$ are switched by the involutions $\rho_{i}$. Under the change $e_{i} \mapsto c_{i} e_{i}$, we have $\lambda_{i} \mapsto\left(\bar{c}_{1} / c_{2}\right) \lambda_{i}$. So again, we could arrange either $\lambda_{2}=1$ or (1.8).

The involutions $\rho_{i}$ act on the set of complex lines through the origin in $\mathbf{C}^{n}$. For $n=2$, this is the Riemann sphere, on which each $\rho_{i}$ fixes the points of a circle $K_{i}$. In case (i) $K_{1} \cap K_{2}$ is two points representing the two cutting lines. In case (ii) the $K_{i}$ are disjoint. If they are taken concentric, centered at 0 , then 0 and $\infty$ represent the two switched lines.

b) Next we consider the real Lagrangian case. Since $\sigma$ is symplectic, it has eigenvalue $\mu^{-1}$ along with $\mu$; we assume $\mu \neq \pm 1$. The above reasoning gives four eigenvalues and vectors,

$$
\sigma e_{1}=\mu e_{1}, \quad \sigma e_{2}=\mu^{-1} e_{2}, \quad \sigma e_{3}=\bar{\mu}^{-1} e_{3}, \quad \sigma e_{4}=\bar{\mu} e_{4}
$$


We assume the vectors normalized by $\omega\left(e_{1}, e_{2}\right)=1, \omega\left(e_{3}, e_{4}\right)=1$, and $\omega\left(e_{i}, e_{j}\right)=0$ otherwise. Then from (1.9) we get

$$
\begin{array}{ll}
\rho_{i} e_{1}=\lambda_{i} e_{3}, & \rho_{i} e_{3}=\bar{\lambda}_{i}^{-1} e_{1}, \\
\rho_{i} e_{2}=-\lambda_{i}^{-1} e_{4}, & \rho_{i} e_{4}=-\bar{\lambda}_{i} e_{2}, \quad \mu=\overline{\lambda_{2} / \lambda_{1}} .
\end{array}
$$

By scaling the eigenvectors while preserving the $\omega\left(e_{i}, e_{j}\right)$, we can again arrange $\lambda_{2}=1$ or (1.8). This is the basic 4-fold case.

There are two special (2-fold) cases,

$$
\text { (i) } \mu=\bar{\mu}^{-1}, \quad(i i) \mu=\bar{\mu},
$$

in which we again assume one-dimensional eigenspaces. In case (i)

$$
\begin{array}{lll}
\sigma e_{1}=\mu e_{1}, & \sigma e_{2}=\mu^{-1} e_{2}, & \mu=\lambda_{1} \bar{\lambda}_{2}, \\
\rho_{i} e_{1}=\lambda_{i} e_{1}, & \rho_{i} e_{2}=-\lambda_{i}^{-1} e_{2}, & \lambda_{i} \bar{\lambda}_{i}=1, i=1,2 .
\end{array}
$$

Again we can achieve (1.8) by scaling.

In case (ii)

$$
\begin{aligned}
& \sigma e_{1}=\mu e_{1}, \quad \sigma e_{2}=\mu^{-1} e_{2}, \quad \mu=\lambda_{2} / \lambda_{1} \\
& \rho_{i} e_{1}=\lambda_{i} e_{2}, \quad \rho_{i} e_{2}=\lambda_{i}^{-1} e_{1}, \quad \lambda_{i}=\bar{\lambda}_{i}, i=1,2
\end{aligned}
$$

the latter following from (1.7). This time the change $e_{i} \mapsto c_{i} e_{i}, c_{1} c_{2}=1$ results in $\lambda_{i} \mapsto\left|c_{1}\right|^{2} \lambda_{i}$. The change $\left(e_{1}, e_{2}\right) \mapsto\left(e_{2},-e_{1}\right)$ results in $\mu \mapsto \mu^{-1}$ and $\lambda_{i} \mapsto-\lambda_{i}^{-1}$. Thus, it is possible to change the sign of $\lambda_{2}$, but the sign of $\lambda_{1} \lambda_{2}$, which by (1.14) is the sign of $\mu$, is invariant. It follows that we can achieve either $\lambda_{2}=1$, or

$$
\lambda_{1} \lambda_{2}=\epsilon \equiv \pm 1, \quad \epsilon=\operatorname{sgn}(\mu) .
$$

In this last case assume that $n=2$ and take coordinates relative to the above determined basis: $z=z_{1} e_{1}+z_{2} e_{2}$. Then

$$
\sigma(z)=\left(\mu z_{1}, \mu^{-1} z_{2}\right), \rho_{i}(z)=\left(\lambda_{i}^{-1} \bar{z}_{2}, \lambda_{i} \bar{z}_{1}\right), i=1,2 .
$$

It follows that the complex curve $z_{1} z_{2}=c$ is invariant under both $\rho_{i}$ if $c=\bar{c}$. Then

$$
\left\{z_{1} z_{2}=c\right\} \cap F P\left(\rho_{i}\right)=\left\{z_{1} z_{2}=c, z_{1} \bar{z}_{1}=c / \lambda_{i}\right\}, i=1,2 .
$$

Thus $z_{1} z_{2}=c$ contains an analytic annulus bounding on $M_{1} \cup M_{2}$, if both $c / \lambda_{i}>0$. This is possible if $\epsilon=+1$. Then taking $c$ 's with $c / \lambda_{1}>0$ gives a one-parameter family of such annuli $A_{c}$ lying on the 3-dimensional algebraic $\operatorname{set} \operatorname{Im}\left(z_{1} z_{2}\right)=0$. As $c$ varies the bounding circles of $A_{c}$ sweep out $M_{1}$ and $M_{2}$, minus the origin, while the $A_{c}$ themselves sweep out a 3 -dimensional manifold contained in the polynomial hull of $M_{1} \cup M_{2}$ ( see also [12]).

c) The complex-linearly reversible case is similar. Now we have (1.10) with

$$
\tau e_{1}=e_{2}, \quad \tau e_{3}=e_{4} .
$$

From (0.4) we get

$$
\begin{aligned}
& \rho_{1}\left(e_{1}, e_{2}, e_{3}, e_{4}\right)=\left(\lambda_{1} e_{3}, \lambda_{2} e_{4}, \bar{\lambda}_{1}^{-1} e_{1}, \bar{\lambda}_{2}^{-1} e_{2}\right), \\
& \rho_{2}\left(e_{1}, e_{2}, e_{3}, e_{4}\right)=\left(\lambda_{2} e_{3}, \lambda_{1} e_{4}, \bar{\lambda}_{2}^{-1} e_{1}, \bar{\lambda}_{1}^{-1} e_{2}\right) .
\end{aligned}
$$


A change $e_{i} \mapsto c_{i} e_{i}$ with $c_{1}=c_{2}$ and $c_{3}=c_{4}$ preserves (1.10) and allows us to achieve (1.8).

We may again have the two 2-fold cases (1.12). In case (i) we have

$$
\begin{gathered}
\rho_{1}\left(e_{1}, e_{2}\right)=\left(\lambda_{1} e_{1}, \lambda_{2} e_{2}\right), \quad \rho_{2}\left(e_{1}, e_{2}\right)=\left(\lambda_{2} e_{1}, \lambda_{1} e_{2}\right), \\
\left|\lambda_{i}\right|=1, \quad \mu=\lambda_{1} \bar{\lambda}_{2},
\end{gathered}
$$

and a change of basis will give (1.8). In case (ii)

$$
\rho_{i}\left(e_{1}, e_{2}\right)=\left(\lambda_{i} e_{2}, \bar{\lambda}_{i}^{-1} e_{1}\right), \quad \bar{\lambda}_{1} \lambda_{2}=1, \quad \mu=\overline{\lambda_{2} / \lambda_{1}}
$$

A change of basis as above allows us to get $\lambda_{1}>0$, and hence (1.8). We refer to [5] for more details on the holomorphically reversible case.

2. Non-linear involutions. We consider a pair of non-linear anti-holomorphic involutions $\rho_{1}, \rho_{2}$ and the anti-reversible map $\sigma$, which may be given by either convergent or formal power series at the orgin of $\mathbf{C}^{n}$. We shall assume that the results of the previous section have been applied to the linear parts of these maps as needed, and write

$$
\begin{aligned}
\sigma(z) & =M z+S(z), \quad S(z)=\sum_{|J| \geq 2} b_{J} z^{J}, \\
\rho_{i}(z) & =L_{i} \bar{z}+R_{i}(z), \quad R_{i}(z)=\sum_{|J| \geq 2} c_{J} \bar{z}^{J}, \quad i=1,2 .
\end{aligned}
$$

Writing out the relations $\sigma=\rho_{1} \rho_{2}$ and $\rho_{i}^{2}=I$ gives

$$
\begin{aligned}
S=L_{1} \bar{R}_{2}+R_{1} \circ \rho_{2}, \quad M & =L_{1} \bar{L}_{2}, I=L_{i} \bar{L}_{i}, \\
0 & =L_{1} \bar{R}_{1}+R_{1} \circ \rho_{1}, \quad 0=L_{2} \bar{R}_{2}+R_{2} \circ \rho_{2} .
\end{aligned}
$$

To eliminate $R_{2}$ and $\bar{R}_{2}$, mutliply the first equation on the left by $\bar{L}_{1}$, and substitute this and its conjugate into the third equation. Then conjugation, left multiplication by $L_{1}$, and using the first equation of the second line gives the first of the following relations.

$$
\begin{aligned}
R_{1}-M R_{1} \circ \sigma & =S \circ \rho_{2}+M L_{1} \bar{S}, \\
R_{2} \circ \sigma^{-1}-M R_{2} & =S \circ \rho_{2}+L_{1} \bar{S} \circ \sigma^{-1} .
\end{aligned}
$$

The second equation is similarly derived.

As a first application in the generic case, suppose that $\sigma$ has already been linearized,

$$
\sigma(z)=M z, \quad M=\operatorname{diag}\left(\mu_{1}, \ldots, \mu_{n}\right) .
$$

By (1.6) the eigenvalues (i) have modulus one, or (ii) occur in pairs. We have zero on the right hand sides of (2.3), and we may compose the second equation with $\sigma$. Substitution of the series in (2.1) into (2.3) gives

$$
\begin{gathered}
\left(1-\bar{\mu}^{J} \mu_{\alpha}\right) c_{i \alpha J}=0,1 \leq \alpha \leq n,|J| \geq 2, i=1,2, \\
\bar{\mu}^{J}=\bar{\mu}_{1}^{j_{1}} \cdots \bar{\mu}_{n}^{j_{n}} .
\end{gathered}
$$


The matrix $M$ is non-resonant, for the full linearization problem, if none of the coefficients of the $c_{i \alpha J}$, i. e. the divisors, in (2.5) vanishes. It then follows that all the coefficients $c_{i \alpha J}$ vanish, and that the $\rho_{i}$ are anti-linear.

More generally, we assume that $\sigma$ admits an invariant complex submanifold $N$, passing through the origin of $\mathbf{C}^{n}$, on which it may or may not be linearizable. As the switched curves show, $N$ may not be left invariant by the $\rho_{i}$; some further conditions are needed.

We choose coordinates

$$
z=\left(z^{\prime}, z^{\prime \prime}\right) \in \mathbf{C}^{l} \times \mathbf{C}^{n-l}
$$

so that $N$ is the linear space $z^{\prime \prime}=0^{\prime \prime}$. Our further assumption is that this splitting is also preserved by the linear parts of the $\rho_{i}$. Then we may write

$$
\sigma(z)=\left(\begin{array}{c}
M^{\prime} z^{\prime}+S^{\prime}\left(z^{\prime}, z^{\prime \prime}\right) \\
M^{\prime \prime} z^{\prime \prime}+S^{\prime \prime}\left(z^{\prime}, z^{\prime \prime}\right)
\end{array}\right), \quad \rho_{i}(z)=\left(\begin{array}{c}
L_{i}^{\prime} \bar{z}^{\prime}+R_{i}^{\prime}\left(z^{\prime}, z^{\prime \prime}\right) \\
L_{i}^{\prime \prime} \bar{z}^{\prime \prime}+R_{i}^{\prime \prime}\left(z^{\prime}, z^{\prime \prime}\right)
\end{array}\right)
$$

We are assuming that $S^{\prime \prime}\left(z^{\prime}, 0^{\prime \prime}\right)=0^{\prime \prime}$, and we want to show that $R_{i}^{\prime \prime}\left(z^{\prime}, 0^{\prime \prime}\right)=0^{\prime \prime}$, $i=1,2$. Taking the "-components in (2.7), restricting to $z^{\prime \prime}=0^{\prime \prime}$, and using the condition on $S^{\prime \prime}$ gives

$$
\begin{aligned}
\left(R_{1}^{\prime \prime}-M^{\prime \prime} R_{1}^{\prime \prime} \circ \sigma\right)_{z^{\prime \prime}=0^{\prime \prime}} & =\left(S^{\prime \prime} \circ \rho_{2}\right)_{z^{\prime \prime}=0^{\prime \prime}}, \\
\left(R_{2}^{\prime \prime} \circ \sigma^{-1}-M^{\prime \prime} R_{2}^{\prime \prime}\right)_{z^{\prime \prime}}=0^{\prime \prime} & =\left(S^{\prime \prime} \circ \rho_{2}\right)_{z^{\prime \prime}=0^{\prime \prime}} .
\end{aligned}
$$

Suppose that $R_{2}^{\prime \prime}\left(z^{\prime}, 0^{\prime \prime}\right)=O\left(\left|z^{\prime}\right|^{k}\right)$. Since $S^{\prime \prime}\left(L_{2} \bar{z}\right)=0^{\prime \prime}$ and $\bar{S}^{\prime \prime}\left(\sigma^{-1}(z)\right)=0^{\prime \prime}$ for $z^{\prime \prime}=0^{\prime \prime}$, the second equation in (2.8) shows that the terms of order $k$ satisfy

$$
R_{2 ; k}^{\prime \prime}\left(M^{\prime-1} z^{\prime}, 0^{\prime \prime}\right)-M^{\prime \prime} R_{2 ; k}^{\prime \prime}\left(z^{\prime}, 0^{\prime \prime}\right)=0^{\prime \prime}
$$

It follows that if we have the non-resonance conditions

$$
\bar{\mu}^{\prime-J}-\mu_{\alpha}=\bar{\mu}_{1}^{-j_{1}} \cdots \bar{\mu}_{l}^{-j_{l}}-\mu_{\alpha} \neq 0, \quad j_{1}+\cdots j_{l} \geq 2, i<\alpha \leq n
$$

then we must have $R_{2 ; k}^{\prime \prime}\left(z^{\prime}, 0^{\prime \prime}\right)=0^{\prime \prime}$; and by induction $R_{2}^{\prime \prime}\left(z^{\prime}, 0^{\prime \prime}\right)=0^{\prime \prime}$. A similar argument with the first equation in $(2.8)$ now shows that $R_{1}^{\prime \prime}\left(z^{\prime}, 0^{\prime \prime}\right)=0^{\prime \prime}$.

Suppose that, in addition, $\sigma$ is linear on the invariant submanifold; that is $S^{\prime}\left(z^{\prime}, 0^{\prime \prime}\right)=0^{\prime}$ and $S^{\prime \prime}\left(z^{\prime}, 0^{\prime \prime}\right)=0^{\prime \prime}$. Then the previous arguments show that $R_{i}^{\prime \prime}\left(z^{\prime} .0^{\prime \prime}\right)=0^{\prime \prime}$, and $R_{i}^{\prime}\left(z^{\prime} .0^{\prime \prime}\right)=0^{\prime}$, provided that we have the stronger non-resonance conditions

$$
\bar{\mu}^{\prime-J}-\mu_{a}=\bar{\mu}_{1}^{-j_{1}} \cdots \bar{\mu}_{l}^{-j_{l}}-\mu_{a} \neq 0, \quad j_{1}+\cdots j_{l} \geq 2, \quad 1 \leq a \leq n .
$$

This proves the following.

Proposition 1. Suppose that $\sigma$ and the linear parts of $\rho_{1}$ and $\rho_{2}$ are as in (2.7), leaving invariant the complex submanifold $z^{\prime \prime}=0^{\prime \prime}$.

a) If the conditions (2.10) hold, then $\rho_{1}$ and $\rho_{2}$ also leave $z^{\prime \prime}=0^{\prime \prime}$ invariant.

b) If $\sigma$ is also linear on $z^{\prime \prime}=0^{\prime \prime}$, and conditions (2.11) hold, then $\rho_{1}$ and $\rho_{2}$ are anti-linear on $z^{\prime \prime}=0^{\prime \prime}$. 
3. Results on the map $\sigma$. The formal arguments of the last section reduce some problems about the pair of involutions $\rho_{i}$ to properties of the map $\sigma$, which we now take up.

We assume that $\sigma$ has the form in (2.1), so that the linear part of $\sigma$ preserves $z^{\prime \prime}=0^{\prime \prime}$. We want to find an $l$-dimensional $\sigma$-invariant submanifold tangent to $z^{\prime \prime}=0^{\prime \prime}$ at 0 . It will be given as the image of a map $f: \mathbf{C}^{l} \rightarrow \mathbf{C}^{n}$,

$$
f(w)=\left(\begin{array}{c}
w+F^{\prime}(w) \\
0+F^{\prime \prime}(w)
\end{array}\right), \quad F(w)=\sum_{|J| \geq 2} c_{J} w^{J}
$$

with an induced map $\hat{\sigma}$ on $\mathbf{C}^{l}$,

$$
\hat{\sigma}(w)=M^{\prime} w+\hat{S}(w) .
$$

Writing out the functional equation $\sigma \circ f=f \circ \hat{\sigma}$ gives

$$
\begin{aligned}
F^{\prime}(\hat{\sigma}(w))-M^{\prime} F^{\prime}(w) & =S^{\prime}(f(w))-\hat{S}(w) \\
F^{\prime \prime}(\hat{\sigma}(w))-M^{\prime \prime} F^{\prime \prime}(w) & =S^{\prime \prime}(f(w)) .
\end{aligned}
$$

For the problem of an invariant submanifold with linearization, $\hat{S}=0$, we have, on the lefthand sides of (3.3), the divisors

$$
\mu^{\prime J}-\mu_{a}=\mu_{1}^{j_{1}} \cdots \mu_{l}^{j_{l}}-\mu_{a}, \quad 1 \leq a \leq n,|J| \geq 2 .
$$

If these are all non-zero, then a unique formal solution $F$ exists. To state sufficient conditions for convergence of the type given by A. D. Brjuno [2], [7], we define

$$
\omega(m)=\min \left\{\left|\mu^{\prime J}-\mu_{a}\right|: 1 \leq a \leq n, 2 \leq|J| \leq m\right\},
$$

and choose an increasing sequence of integers $1=q_{0}<q_{2}<\cdots<q_{j}<\cdots$, say $q_{j}=2^{j}$. Brjuno's condition is then

$$
\sum_{j=0}^{\infty}-q_{j}^{-1} \log \omega\left(q_{j+1}\right)<\infty .
$$

By a theorem of Pöschel [7] the condition (3.6) implies that the power series solution $f$ has some positive radius of convergence. result.

By combining these considerations on $\sigma$ with proposition (1b), we get the following

THEOREM 2. Suppose that $\sigma=\rho_{1} \rho_{2}$, where the (convergent) maps are as in (2.7), and the linear part of $\sigma$ satisfies (2.4), (2.11), (3.5), and (3.6). Then there exists an l-dimensional analytic submanifold $N$ tangent to the space $z^{\prime \prime}=0^{\prime \prime}$ at 0 in $\mathbf{C}^{n}$, which is invariant by $\sigma, \rho_{1}$, and $\rho_{2}$. These maps are simultaneously holomorphically (anti)linearizable on $N$.

For the existence of a cutting curve, we take $l=1$, and $\left|\mu_{1}\right|=1$ in the theorem. This is related to Klingenberg's construction of asymptotic curves [4]. Even in the case $n=1$, we do not know if the Brjuno condition is necessary for the result, i. e. whether an analogue of Yoccoz's theorem [14] holds in the anti-reversible category.

For the existence of switched curves, we take $l=2$ and $\mu_{2}=\bar{\mu}_{1}^{-1} \neq \mu_{1}$, thus

$$
\mu_{1}=r e^{i \theta}, \mu_{2}=r^{-1} e^{i \theta}, \mu_{\alpha}=r_{\alpha} e^{i \theta_{\alpha}}, 3 \leq \alpha \leq n,
$$


with $r>1$. We may simplify and sharpen the conditions for convergence. Since all positive or negative powers of $r$ will be bounded away from the $r_{\alpha}$, we need only consider $a=1,2$, and $\left|j_{1}-j_{2}\right|=1$ in (2.11) and (3.4). A simple consideration of all cases shows that we may replace (3.5) by

$$
\omega(m)=\min \left\{\left|e^{2 j \theta i}-1\right|: 1 \leq j \leq m\right\} .
$$

Of course, the theorem gives linearization on a 2-dimensional submanifold, which is a-priori stronger than just the existence of a pair of switched curves.

For the invariant submanifold problem without linearization, the question of convergence is much more delicate. Following [9] and [5] we consider a special situation, which we shall need later. We assume $l=2$ in (2.6) and

$$
M^{\prime}=\operatorname{diag}\left(\mu_{1}, \mu_{2}\right), \quad \mu_{1} \mu_{2}=1
$$

and set

$$
F=\left(F^{\prime}, F^{\prime \prime}\right)=\sum c_{j_{1} j_{2}} w_{1}^{j_{1}} w_{2}^{j_{2}}, F^{\prime}=\left(F_{1}, F_{2}\right), F^{\prime \prime}=\left(F_{\alpha}\right)
$$

We assume that the terms of degree $<k$ in $F$ and in $\hat{S}=\left(\hat{S}_{1}, \hat{S}_{2}\right)$ have been determined, and analyze the as yet undetermined terms of order $k$ in (3.3). We find the divisors

$$
\mu_{1}^{k-2 j}-\mu_{1}, \mu_{1}^{k-2 j}-\mu_{1}^{-1}, \mu_{1}^{k-2 j}-\mu_{\alpha}
$$

for the coefficients of $F_{1}, F_{2}$, and $F_{\alpha}$, respectively. The first two vanish for $k=2 j+1$ and $k=2 j-1$, respectively, and we assume that all the other divisors are non-zero. Then $\hat{S}$ is uniquely determined at $k$-th order to make up for the lost terms. Also, we normalize $F$ to make it unique.

More precisely, we introduce the "type" of a power series: type $\left(w_{1}^{j_{1}} w_{2}^{j_{2}}\right)=j_{1}-j_{2}$, and write for $k \in \mathbf{Z}$

$$
[F]_{k}=\sum_{j_{1}-j_{2}=k} c_{j_{1} j_{2}} w_{1}^{j_{1}} w_{2}^{j_{2}}
$$

Then we require

$$
\left[F_{1}\right]_{+1}=0, \quad\left[F_{2}\right]_{-1}=0, \quad \hat{S}_{1}=\left[\hat{S}_{1}\right]_{+1}, \quad \hat{S}_{2}=\left[\hat{S}_{2}\right]_{-1} .
$$

It follows that there is a unique so normalized formal map $f$ satisfying $\sigma \circ f=f \circ \hat{\sigma}$ and

$$
\hat{\sigma}(w)=\left(\mu(t) w_{1}, \nu(t) w_{2}\right), \quad t=w_{1} w_{2},
$$

where $\mu(0)=\mu_{1}, \nu(0)=\mu_{2}$, are the given eigenvalues.

In general, there is no convergence argument for $f$, even if $\sigma$ is convergent [9]. However, if $\sigma$ is convergent, and if

$$
\mu(t) \nu(t)=1, \quad\left|\mu_{1}\right| \neq 1,
$$

where the first is a formal power series relation, then $f$ does converge. In fact, the functional equation (3.3) is now of the form of (4.1) in [5], with $\xi=w_{1}, \eta=w_{2}$, and $\zeta=0$. The powers of $\mu_{1}$ and $\mu_{1}^{-1}$ tend to 0 or $\infty$, and we assume that none equals 
any $\mu_{\alpha}$. Then there are no small divisors, and the argument proving theorem (4.1) in [5] applies directly to give convergence here. The case $l=n=2$ is in [9].

The condition (3.15) may be interpreted as saying that the map $\sigma$ is "integrable", since it implies that the function $w_{1} w_{2}$ is $\sigma$-invariant.

The foregoing can be applied, if the map $\sigma$ is symplectic, by the following.

Proposition 2. Let the holomorphic symplectic map $\sigma$ have the form (2.6), (2.7), (2.4) with $l=2$, and eigenvalues satisfying $\mu_{1} \mu_{2}=1,\left|\mu_{1}\right| \neq 1$, and no integral power of $\mu_{1}$ equal to any $\mu_{\alpha}$. Suppose that the $\left(z_{1}, z_{2}\right)$-plane is symplectic at the origin. Then the formal transformation $f$ just described converges, giving an invariant 2-manifold on which $\sigma$ corresponds to the Birkhoff normal form map

$$
\hat{\sigma}(w)=\left(\mu(t) w_{1}, \mu(t)^{-1} w_{2}\right), \quad t=w_{1} w_{2}, \quad \mu(0)=\mu_{1} .
$$

For the proof it suffices to verify the first condition in (3.15). From $\sigma^{*} \omega=\omega$ and $\sigma \circ f=f \circ \hat{\sigma}$, we see that the 2 -form $f^{*} \omega$ is $\hat{\sigma}^{*}$-invariant. To simplify notation we denote it by $\omega$ and work on $\mathbf{C}^{2}$. By our assumptions,

$$
\omega=a(w) d w_{1} \wedge d w_{2}, \quad a(0) \neq 0 .
$$

From (3.14) the substitution $\hat{\sigma}^{*} \omega=\omega$ gives

$$
a\left(\mu(t) w_{1}, \nu(t) w_{2}\right) \varphi^{\prime}(t)=a\left(w_{1}, w_{2}\right), \quad \varphi(t)=\mu(t) \nu(t) t .
$$

We have $\varphi^{\prime}(0)=1$. By taking the part of type 0 in (3.18), we get an equation $A(\varphi(t)) \varphi^{\prime}(t)=A(t), A(0) \neq 0$. Integrating, $B^{\prime}(t)=A(t), B(0)=0$, gives

$$
B(\varphi(t))=B(t)=\sum_{j=1}^{\infty} b_{j} t^{j}, b_{1} \neq 0 .
$$

If $\varphi(t)=t+c_{k} t^{k}+\cdots$, then substitution into (3.19) gives $b_{k}=b_{1} c_{k}+b_{k}$. Hence, $c_{k}=0$, and so $\varphi(t) \equiv t$, which proves (3.15).

The analogue of this result for flows is already in [9].

4. Anti-reversible symplectic maps in $\mathbf{C}^{2}$. We take $w=\left(w_{1}, w_{2}\right) \in \mathbf{C}^{2}$ with $\omega=d w_{1} \wedge d w_{2}$, and $\rho_{1}, \rho_{2}, \sigma$ satisfying (0.5). We assume that $\sigma$ is in normal form (3.16), and derive the form of $\rho_{1}$ and $\rho_{2}$, in the two cases (1.12), using the formulae (2.3). In (2.1) we denote the two components by $S=\left(S^{\prime}, S^{\prime \prime}\right)$ and $R_{i}=\left(R_{i}^{\prime}, R_{i}^{\prime \prime}\right)$.

In case (i) the second equation in (2.3) is

$$
\begin{aligned}
R_{2}^{\prime} \circ \sigma^{-1}-\mu_{1} R_{2}^{\prime} & =S^{\prime} \circ \rho_{2}+\lambda_{1} \bar{S}^{\prime} \circ \sigma^{-1}, \\
R_{2}^{\prime \prime} \circ \sigma^{-1}-\mu_{1}^{-1} R_{2}^{\prime \prime} & =S^{\prime \prime} \circ \rho_{2}-\lambda_{1}^{-1} \bar{S}^{\prime \prime} \circ \sigma^{-1} .
\end{aligned}
$$

We use the type decomposition (3.12), but with respect to the conjugate variables $\left(\bar{w}_{1}, \bar{w}_{2}\right)$. Since $\left[Q \circ \sigma^{-1}\right]_{m}=\overline{\mu(t)}^{-m}[Q]_{m}$, multiplying through by $\overline{\mu(t)}^{m}$ gives

$$
\begin{aligned}
\left(1-\mu_{1} \overline{\mu(t)}^{m}\right)\left[R_{2}^{\prime}\right]_{m}=\overline{\mu(t)}^{m}\left[S^{\prime} \circ \rho_{2}\right]_{m}, \quad m \neq+1, \\
\left(1-\mu_{1}^{-1} \overline{\mu(t)}^{m}\right)\left[R_{2}^{\prime \prime}\right]_{m}=\overline{\mu(t)}\left[S^{\prime \prime} \circ \rho_{2}\right]_{m}, \quad m \neq-1,
\end{aligned}
$$

because of the form (3.16) of $\sigma$.

We assume that $\left[R_{2}^{\prime}\right]_{m}, m \neq+1$, and $\left[R_{2}^{\prime \prime}\right]_{m}, m \neq-1$ have no terms of order $<k$. Since $S \circ \rho_{2}(z)=S\left(L_{2} \bar{z}\right)+O\left(R_{2}\right)$, there are no terms of order $k$ on the right hand 
side. Since $1-\mu_{1} \bar{\mu}_{1}^{m} \neq 0$, if $m \neq+1$, and $1-\mu_{1}^{-1} \bar{\mu}_{1}^{m} \neq 0$, if $m \neq-1$, it follows that the terms of order $k$ vanish. Thus, $\rho_{2}$ has the form

$$
\rho_{2}(w)=\left(\lambda_{2}(\bar{t}) \bar{w}_{1}, \kappa_{2}(\bar{t}) \bar{w}_{2}\right) .
$$

A similar but simpler argument with the first equation in (4.1) shows that

$$
\rho_{1}(w)=\left(\lambda_{1}(\bar{t}) \bar{w}_{1}, \kappa_{1}(\bar{t}) \bar{w}_{2}\right) .
$$

Using (4.3), (4.4) in the conditions $\rho_{i}^{*} \omega=-\bar{\omega}$ gives $\partial_{\bar{t}}\left(\lambda_{i}(\bar{t}) \kappa_{i}(\bar{t}) \bar{t}\right)=-1$. Hence $\lambda_{i}(\bar{t}) \kappa_{i}(\bar{t})=-1$, and

$$
\begin{aligned}
\rho_{i}(w) & =\left(\lambda_{i}(\bar{t}) \bar{w}_{1},-\lambda_{i}(\bar{t})^{-1} \bar{w}_{2}\right), \quad i=1,2 \\
\mu(t) & =\lambda_{1}(-t) \bar{\lambda}_{2}(t) .
\end{aligned}
$$

Similarly, the condition $\rho_{i}^{2}=I$ gives

$$
\lambda_{i}(t)=\lambda_{i}^{*}(t), \quad \lambda_{i}^{*}(t) \equiv 1 / \bar{\lambda}_{i}(-t),
$$

where the bar means to conjugate the coefficients, and $*$ is an involutive multiplicative homomorphism.

For further simplification, we conjugate by a transformation

$$
f(w)=\left(\alpha(t) w_{1}, \alpha(t)^{-1} w_{2}\right)
$$

which preserves $\omega$ and commutes with $\sigma$. This preserves the form (4.5) and results in

$$
\lambda_{i}(t) \mapsto \lambda_{i}(t) \beta(t) \beta^{*}(t), \quad i=1,2,
$$

where $\beta(t)=\alpha(-t)$. If we choose $\beta(t)=\lambda_{2}(t)^{-1 / 2}$, or $\beta(t)=\left(\lambda_{1}(t) \lambda_{2}(t)\right)^{-1 / 2}$, the properties of $*$ show that we get, respectively $\lambda_{2}(t)=1$, or

$$
\lambda_{1}(t) \lambda_{2}(t)=1 \text {. }
$$

Now we turn to case (ii). The equations (4.1) are now replaced by

$$
\begin{aligned}
R_{2}^{\prime} \circ \sigma^{-1}-\mu_{1} R_{2}^{\prime} & =S^{\prime} \circ \rho_{2}+\lambda_{1}^{-1} \bar{S}^{\prime \prime} \circ \sigma^{-1}, \\
R_{2}^{\prime \prime} \circ \sigma^{-1}-\mu_{1}^{-1} R_{2}^{\prime \prime} & =S^{\prime \prime} \circ \rho_{2}+\lambda_{1} \bar{S}^{\prime} \circ \sigma^{-1} .
\end{aligned}
$$

We now have (4.2) with $m \neq-1$ in the first equation and $m \neq+1$ in the second. The same arguments give

$$
\rho_{i}(w)=\left(\lambda_{i}(\bar{t}) \bar{w}_{2}, \kappa_{i}(\bar{t}) \bar{w}_{1}\right)
$$

Again the conditions $\rho_{i}^{*} \omega=-\bar{\omega}$ and $\rho_{i}^{2}=I$ give

$$
\begin{aligned}
\rho_{i}(w) & =\left(\lambda_{i}(\bar{t}) \bar{w}_{2}, \lambda_{i}(\bar{t})^{-1} \bar{w}_{1}\right), \quad \bar{\lambda}_{i}(t)=\lambda_{i}(t), \quad i=1,2 . \\
\mu(t) & =\lambda_{1}(t) / \lambda_{2}(t) .
\end{aligned}
$$

Conjugation by $f$ in (4.7) again preserves the form (4.12) and results in

$$
\lambda_{i}(t) \mapsto \lambda_{i}(t)(\alpha(t) \bar{\alpha}(t))^{-1}, \quad \lambda_{1}(t) \lambda_{2}(t) \mapsto \lambda_{1}(t) \lambda_{2}(t)(\alpha(t) \bar{\alpha}(t))^{-2} .
$$


To preserve the reality condition in (4.12), we restrict to $\alpha(t)=\bar{\alpha}(t)$. The symplectic change of coordinates $\left(w_{1}, w_{2}\right) \mapsto\left(w_{2},-w_{1}\right)$ results in

$$
\mu(t) \mapsto \mu(-t)^{-1}, \quad \lambda_{i}(t) \mapsto-\lambda_{i}(-t)^{-1} .
$$

By extracting a real square root or fourth root, we can achieve either $\lambda_{2}(t)=1$, or

$$
\lambda_{1}(t) \lambda_{2}(t)=\epsilon= \pm 1, \quad \epsilon=\operatorname{sgn}\left(\mu_{1}\right) .
$$

If we restrict to the surface $M_{i}=F P\left(\rho_{i}\right)$ in case (ii), then $w=\rho_{i}(w)$, and (4.12) gives $w_{2}=\lambda_{i}(\bar{t})^{-1} \bar{w}_{1}$, and $\lambda_{i}(\bar{t})=\lambda_{i}(t)$. If we multiply the second component of (4.12) by $w_{1}$ and set $s=\left|w_{1}\right|^{2}$, then we get $s=t \lambda_{i}(t)$, which is an invertible power series with real coefficients. It follows that $t=w_{1} w_{2}$ is real when restricted to $M_{i}$. Let $t=s \phi_{i}(s)$ be the inverse function, and define a real function of $s$ by

$$
r_{i}(s)=\int_{0}^{s} \lambda_{i}\left(s \phi_{i}(s)\right)^{-1} d s .
$$

This results in the following equation for $M_{i}$

$$
M_{i}: w_{2}=\partial_{w_{1}} r_{i}, \quad r_{i}=r_{i}\left(\left|w_{1}\right|^{2}\right),
$$

which is precisely $(0.6),(0.7),(0.8)$. This also anticipates the developments of the next section.

As in section 1 we consider the complex curve $w_{1} w_{2}=c$, for small real $c$. It is invariant under both $\rho_{1}$ and $\rho_{2}$, and

$$
\left\{w_{1} w_{2}=c\right\} \cap F P\left(\rho_{i}\right)=\left\{w_{1} w_{2}=c,\left|w_{1}\right|^{2}=c \lambda_{i}(c)\right\} .
$$

If $\epsilon=+1$, we choose $c$ so that $c \lambda_{1}(c)>0$. Then

$$
c \lambda_{1}(c) \leq\left|w_{1}\right|^{2} \leq c \lambda_{2}(c), \quad w_{2}=c / w_{1},
$$

defines an analytic annulus $A_{c}$ on the curve with boundary on $M_{1} \cup M_{2}$, which shrinks to the origin as $c \rightarrow 0$. The annular modulus (ratio of inner to outer radius) is $\left|\lambda_{1}(c)\right|$, which approaches $\left|\lambda_{1}(0)\right| \neq 0$. These annuli $A_{c}$ sweep out a three dimensional manifold lying on the real analytic levi-flat set $\operatorname{Im}\left(w_{1} w_{2}\right)=0, \operatorname{Re}\left(w_{1} w_{2}\right)>0$.

In case (i) the transformation of $\sigma$ into the normal form (3.16) exists if the linear part of $\sigma$ is non-resonant, but may not converge [9]. In case (ii) it always exists and converges [9]. Combining this with the arguments just given yields the following.

THEOREM 3. Let $M_{1}$ and $M_{2}$ be a pair of analytic real Lagrangian surfaces in $\mathbf{C}^{2}$, intersecting transversely at the orgin. Let $\rho_{1}$ and $\rho_{2}$ be the associated antiholomorphic involutions, with linear parts satisfying (1.13) in case (i), or (1.14) in case (ii). Suppose the linear part of $\sigma=\rho_{1} \rho_{2}$ is non-resonant. Then in case (i) there exists a formal symplectic transformation taking the pair $\rho_{i}$ into the form (4.5). In case (ii) there exists a holomorphic symplectic transformation taking the $\rho_{i}$ into the form (4.12) .

By combining proposition (2) and theorem (3), we may carry some of the above results over to the higher dimensional case. For example, we have the following result.

THEOREM 4. Let $M_{1}$ and $M_{2}$ be a pair of analytic real Lagrangian surfaces in $\mathbf{C}^{2 n}$, with symplectic form (0.5), intersecting transversely at the orgin. Suppose that 
$\sigma$ satisfies the conditions of proposition (2) with $\mu_{1}>0$. Suppose that $\rho_{1}, \rho_{2}$ satisfy the conditions of proposition (2a). Then there exists a real analytic one-parameter family $A_{c}, c_{0}>c>0$, of analytic annuli in $\mathbf{C}^{2 n}$ bounding on $M_{1} \cup M_{2}$, and shrinking to the intersection point as $c \rightarrow 0$.

This family of analytic annuli contributes to the local holomorphic hull of $M_{1} \cup M_{2}$.

5. Real Lagrangians and generating functions. As in [10] we work on the holomorphic cotangent bundle with its canonical structure,

$$
T^{*}\left(\mathbf{C}^{n}\right) \cong \mathbf{C}^{2 n} \ni(z, p), \quad \theta=p \cdot d z=\sum_{\alpha=1}^{n} p_{\alpha} d z_{\alpha}, \quad \omega=d \theta .
$$

Every real-valued function $r(z)$ on $\mathbf{C}^{n}$ gives a real Lagrangian $M \subset T^{*}\left(\mathbf{C}^{n}\right)$, namely the graph of $\partial r$,

$$
M: R_{\alpha} \equiv p_{\alpha}-\partial_{\alpha} r=0, \partial_{\alpha}=\partial / \partial z_{\alpha}, \quad 1 \leq \alpha \leq n,
$$

since restricting to $M$ gives $\operatorname{Re}(\omega)=d \operatorname{Re}(\theta)=d(\partial r+\bar{\partial} r) / 2=0 . \quad \mathrm{A}(1,0)$-vector $(d z, d p)$ is tangent to $M$, if it satisfies

$$
\partial R_{\alpha}=0, \partial \bar{R}_{\alpha}=-\sum_{\beta} \partial_{\beta} \partial_{\bar{\alpha}} r d z_{\beta}=0
$$

This means that its projection $d z$ is in the nullspace of the Levi form, or (1,1)-hessian of $r$. In particular, $M$ is totally real if and only if $r$ has non-degenerate Levi form. All real analytic, totally real, real Lagrangians $M$ are locally equivalent. This follows from applying the real analytic Darboux theorem to $(M, \operatorname{Im}(\omega))$ and then complexifying back to the ambient space. For a single smooth $M$, we may take $r=z \cdot \bar{z}$, to arbitrarily high order.

Every holomorphic function $h(z)$ generates the symplectic map $\left(z_{\alpha}, p_{\alpha}\right) \mapsto$ $\left(z_{\alpha}, p_{\alpha}+\partial_{\alpha} h\right)$. This transforms $p_{\alpha}=\partial_{\alpha} r$ into $p_{\alpha}=\partial_{\alpha}(r-h-\bar{h})$, and allows us to remove any purely holomorphic and anti-holomorphic terms in $r$. If $M_{1}$ and $M_{2}$ are two real Lagrangians intersecting over $z=0$, then their functions $r_{1}$ and $r_{2}$ have the same linear parts. By so transforming $M_{1}$ and $M_{2}$, we may assume

$$
\begin{aligned}
& r_{1}=\sum_{\alpha, \beta=1}^{n} b_{\alpha \bar{\beta}} z_{\alpha} \bar{z}_{\beta}+2 \operatorname{Re}\left(a_{\alpha \beta} z_{\alpha} z_{\beta}\right)+\cdots, \\
& r_{2}=z \cdot \bar{z}+\cdots, \quad a_{\alpha \beta}=a_{\beta \alpha}, \quad b_{\alpha \bar{\beta}}=\overline{b_{\beta \bar{\alpha}}} .
\end{aligned}
$$

One may apply a linear symplectic transformation to simplify further the quadratic terms in (5.4), and then use a generating function to construct a symplectic transformation to simplify the higher order terms (see [10]). However, this seems to be rather complicated, so we return to the approach of sections 1 and 2 focusing on the involutions $\rho_{i}$ and $\operatorname{map} \sigma$.

We restrict to the case, $n=1$, so that (5.4) gives (0.6). We drop the higher order terms in (0.6) and work with quadratic parts,

$$
\begin{aligned}
& r_{1}=a z^{2}+b z \bar{z}+\overline{a z}^{2}, \quad b \neq 0, \\
& r_{2}=z \bar{z}, \quad(b, a) \neq(1,0) .
\end{aligned}
$$

This gives the linear surfaces

$$
\begin{aligned}
& M_{1}: \quad p=r_{1 z}=2 a z+b \bar{z} \\
& M_{2}: p=r_{2 z}=\bar{z}
\end{aligned}
$$


By solving these equations for $(z, p)$ in terms of $(\bar{z}, \bar{p})$, we get the matrices as in $(2.1)$ for $\rho_{1}, \rho_{2}$, and $\sigma$,

$$
\begin{aligned}
L_{1}= & b^{-1}\left[\begin{array}{cc}
-2 \bar{a} & 1 \\
\Delta & 2 a
\end{array}\right], L_{2}=\left[\begin{array}{ll}
0 & 1 \\
1 & 0
\end{array}\right], M=L_{1} \bar{L}_{2}=b^{-1}\left[\begin{array}{cc}
1 & -2 \bar{a} \\
2 a & \Delta
\end{array}\right] \\
& \operatorname{det}(M-\mu I)=\mu^{2}-2 \delta \mu+1, \quad 2 b \delta=\Delta+1, \Delta=b^{2}-4|a|^{2}
\end{aligned}
$$

For the two cases (1.12) we have: (i) $\mu \bar{\mu}=1$ if and only if $\delta^{2}<1$; and (ii) $\mu=\bar{\mu}$ if and only if $\delta^{2}>1$. The case $\epsilon=+1$ in case (ii) is equivalent to $\delta>1$, since the trace of $M$ must be positive. From this and the results of the last section we readly derive the theorem stated in the introduction.

\section{REFERENCES}

[1] E. BisHop, Differentiable manifolds in complex Euclidean space, Duke Math. J., 32 (1965), pp. $1-22$.

[2] A. D. Bruuno, Analytic form of differential equations, Trans. Moscow Math. Soc., 25 (1971), pp. 131-288, 26 (1972), pp. 199-239.

[3] X. Gong, Anti-holomorphically reversible holomorphic maps that are not holomorphically reversible, to appear.

[4] W. Klingenberg, Asymptotic curves on real analytic surfaces in $\mathbf{C}^{2}$, Math. Ann., 273 (1985), pp. 149-162.

[5] J. Moser AND S. WeBster, Normal forms for real surfaces in $\mathbf{C}^{2}$ near complex tangents and hyperbolic surface transformations, Acta Math., 150 (1983), pp. 255-296.

[6] G. PFEIFFER, On the conformal mapping of curvilinear angles, Trans. AMS, 18 (1917), pp. 185-198.

[7] J. PösCHEL, Invariant manifolds of complex analytic mappings near fixed points, Les Houches, Session XLIII, vol. II (1984), pp. 949-964.

[8] N. SiBONY, personal communication.

[9] C. Siegel AND J. Moser, Lectures on Celestial Mechanics, Springer-Verlag (1971).

[10] S. WeBster, Holomorphic symplectic normalization of a real function, Ann. Sc. Norm. Sup. Pisa, vol. XIX (1992), pp. 69-86.

[11] S. Webster, A note on extremal discs and double valued reflection, AMS. Contemp. Math., 205 (1997), pp. 271-276.

[12] B. WeInstock, On the polynomial convexity of the union of two maximal totally real subspaces of $\mathbf{C}^{n}$, Math. Ann., 282 (1988), pp. 131-138.

[13] H. WeYL, The Classical Groups, Princeton University Press (1946).

[14] J. Yoccoz, Théorème de Siegel, nombres de Bruno et polynômes quadriques, Astérisque, 231 (1995), pp. 3-88. 